\title{
Femur metastasis in carcinoma of the uterine cervix: a rare entity
}

\author{
Giacomo Corrado $\cdot$ Salvatore Santaguida $\cdot$ \\ Gianfranco Zannoni · Giovanni Scambia • \\ Gabriella Ferrandina
}

Received: 12 August 2009 / Accepted: 19 November 2009 / Published online: 2 December 2009

(C) The Author(s) 2009. This article is published with open access at Springerlink.com

\begin{abstract}
Purpose We report the first case of isolated femur metastasis in a locally advanced cervical cancer (LACC) patients.

Case A 40-year-old woman presenting with carcinoma of the uterine cervix, FIGO stage IIb was administered concomitant chemo-radiation and achieved clinical partial response. Before the planned surgery, she developed an isolated metastatic lytic lesion of the left femur. After surgical excision of metastasis, she refused palliative chemotherapy, and radiotherapy, and died 3 months later because of progression of the disease.

Conclusion Bone metastasis is not so infrequent in patients with LACC. Because the prognosis of these patients is poor and most of them die within 1 year after the diagnosis of metastatic disease, the policy of treatment should be directed to maintain their quality of life.
\end{abstract}

Keywords Cervical cancer $\cdot$ Bone metastasis $\cdot$ Femur

G. Corrado $(\bowtie) \cdot$ S. Santaguida · G. Ferrandina

Gynecologic Oncology Unit, Department of Oncology,

Catholic University of the Sacred Heart,

L.go A. Gemelli, 1, 86100 Campobasso, Italy

e-mail: giacomo.corrado@alice.it

G. Zannoni

Institute of Human Pathology,

Catholic University of the Sacred Heart, Rome, Italy

G. Scambia

Gynecologic Oncology Unit,

Catholic University of the Sacred Heart, Rome, Italy

\section{Introduction}

Although the incidence of cervical cancer has been reduced in the developed countries, advanced/recurrent and metastatic disease remains a major cause of cancer death in women. Nearly one-third of patients who present with invasive cervical cancer die of the disease [1].

Cervical cancer patients may develop pelvic recurrence, distant metastases, or a combination of both. A 10-20\% recurrence rate has been reported following primary surgery or radiotherapy in women with stage IB-IIA cervical tumours, while up to $70 \%$ of patients with lymph node metastases or locally advanced tumours relapse. Bone metastases occurred in $16 \%$ of patients, predominantly involving the lumbar and thoracic spine, while are uncommon in the distal appendicular skeleton [2].

We report the first case of bone metastasis from carcinoma of the cervix, wherein the patient developed isolated metastasis to left diaphysis femur.

\section{Case report}

In August 2007, a 40-year-old fertile woman was admitted to the Gynaecologic Oncology Unit of the Catholic University of Rome with a diagnosis of a poorly differentiated epidermoidal carcinoma of the cervix as documented by colposcopy-guided biopsy. Her family and gynaecologic histories were unremarkable.

Gynaecological examination under anaesthesia documented a uterus of normal size, no adnexal masses; the cervix showed an exofitic bleeding lesion $(3 \mathrm{~cm}$ maximum diameter), with obliteration of anterior, lateral and posterior fornices. Biopsy was performed and showed the presence 
of poorly differentiated carcinoma with details compatible with adenosquamous type (Fig. 1a).

Staging work-up included chest X-ray, pelvic ultrasonography (US), and abdomino-pelvic magnetic resonance imaging (MRI) with contrast. Pelvic US documented the presence of a cervical lesion $(52 \times 27 \times 38 \mathrm{~mm})$ richly vascularized at color Doppler scan, that appeared to invade the stroma and the internal uterine orifice. MRI report confirmed the presence of a high-intensity signal area in T2-weighted images lesion in the cervical canal, anteroposterior $47 \mathrm{~mm}$, transversal $52 \mathrm{~mm}$ and cranio-caudal $33 \mathrm{~mm}$ diameter. The left fornix and parametrium were involved, as well as the superior third of vagina. Bilateral iliac and inguinal lymph nodes had pericentimetric diameter. Circulating levels of SCC were $2.6 \mathrm{ng} / \mathrm{ml}$; Ca 125 levels were negative $(20.5 \mathrm{U} / \mathrm{ml})$.

The patient was staged as having FIGO stage IIB cervical cancer, and was triaged to concomitant radio-chemotherapy followed by surgery according to our institutional protocol [3]. Four weeks after the end of concomitant chemoradiotherapy, the patient was evaluated for objective response. Pelvic MRI documented a partial response of all dimensions of the cervical lesion, now measuring $15 \times 18 \times 14 \mathrm{~mm}$.

Two weeks before undergoing the planned surgery, she experienced non-traumatic left diaphysal femur fracture. MRI of left femur showed a complete pathological fracture of the femoral diaphyses with relocation of fragments. At the level of the femur stumps, a voluminous mass involving the median diaphyses was documented (maximum diameter $9 \mathrm{~cm}$ ) (Fig. 2).

In order to exclude the presence of breast and lung cancer, mammography and thorax CT scan were performed. A total body bony scintigraphy was performed and confirmed the presence of the femoral lesion only.

On the basis of FDG-PET findings, a wide surgical excision of the lesion was performed and final pathology revealed the presence of metastasis of poorly differentiated

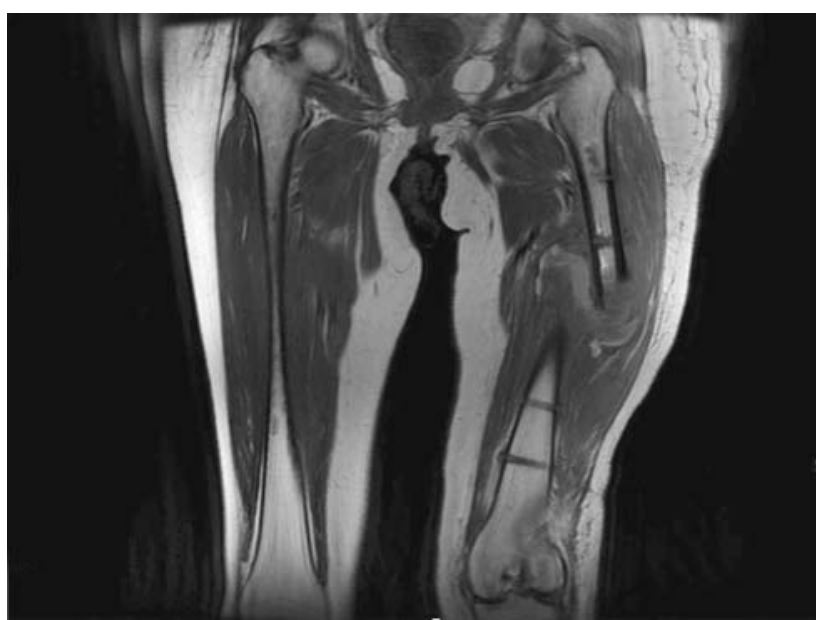

Fig. 2 T2-weighted MRI showing the presence of a complete pathological fracture of the left femoral diaphyses with a voluminous mass of caudal skull diameter of about $9 \mathrm{~cm}$

carcinoma of similar morphology as the primary (Fig. 1b). The surgical margins were free of the disease.

The patient refused palliative chemotherapy and radiotherapy and after 3 months died because of pulmonary embolism.

\section{Discussion}

The occurrence of bone metastases is considered infrequent, likely it is underestimated. Indeed, the rate of the bone metastases in recurrent cervical carcinoma has been shown to increase from 15 to $29 \%$ [4].

Matsuyama et al. [5] reported that the rates of bone recurrence, among 713 patients were $4.0 \%$ in stage I, $6.6 \%$ in stage II, $8.0 \%$ in stage III, and $22.9 \%$ in stage IV. The most frequent site of metastasis was represented by the vertebral column, particularly the lumbar spine (48\%) followed by the pelvic bones. In 32 cases $(67 \%)$, bone lesions
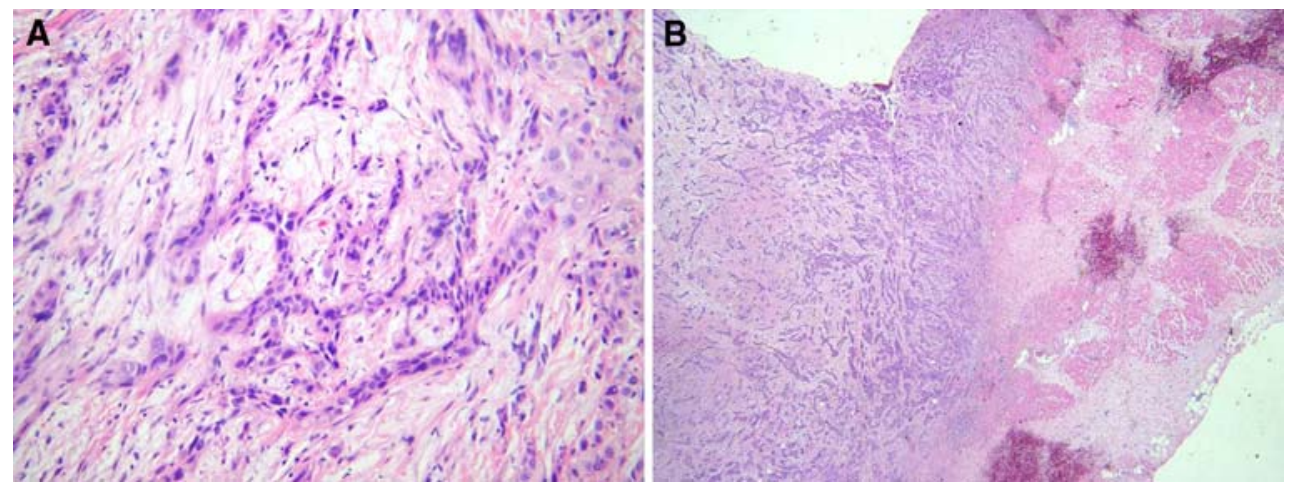

Fig. 1 a Primary cervical carcinoma. Poorly differentiated tumor cells are arranged in cords $(H \& E, \times 200)$. b Metastatic cervical carcinoma. Neoplastic cells infiltrate the scheletric muscle and periosteal tissue $(\mathrm{H} \& \mathrm{E}, \times 50)$ 
were detected within 1 year after completion of the initial treatment, and 36 patients $(75 \%)$ died within 1 year after detection of the metastasis.

Spreading to the bone may occur both by direct extension of the pelvic tumour to the bone, as from soft tissue metastasis outside the pelvis, and least commonly by haematogenous route.

Establishing the diagnosis of femur metastasis sometimes can be difficult: indeed, although femur metastases can be painful or palpable or cause deformity, sometimes they can be asymptomatic.

Diagnostic imaging techniques are useful tools in order to define the site and extension of the disease, and exclude diffuse metastases. In our case FDG-PET findings were critical in order to plan patient management, because a diffuse metastatic disease was excluded and surgical removal of the femur recurrence could be planned.

Once the bone lesion is diagnosed, the treatment is usually directed towards both pain relief and preservation of quality of life considering the patients' short life expectancy. Given the rare occurrence of bone metastasis, there are no specific guidelines on the therapeutic options, which can include radiotherapy, chemotherapy, and surgery according to the clinical setting: indeed, the real benefit of these approaches remains questionable given the very poor prognosis of these patients.

Generally, in case of a solitary and resectable bone metastasis surgical management followed by palliative radiotherapy is recommended [6].

We offered our patient a multimodal approach, considering her young age and good performance status, and the documentation of a single and surgically amenable recurrence.

The acknowledgement of the femur metastasis as a marker of systemic disease prompted us to also plan the administration of chemotherapy: in particular the platinumtaxane combination was chosen on the basis of the high response rate documented with this regimen compared to cisplatin alone [7].

In conclusion, since bone metastasis is not so infrequent, especially in patients with locally advanced cervical cancer (LACC), our data confirm that the total body PET-TC scan should be included in staging work up as recommended by the guidelines of the National Comprehensive Cancer Network (NCCN) [8].

It has to be acknowledged that besides its role in clinical staging pre-treatment as well as post-chemoradiation total body PET-CT scan findings have been shown to accurately predict clinical outcome in LACC [9].

Moreover, very recent data on LACC seem to suggest that PET-CT scan guided treatment can also improve overall survival [10].

Additionally, since the prognosis of these patients is dismal, and most of them died within 1 year after diagnosis of metastatic disease, the policy of treatment should be directed to maintain their quality of life.

\section{Conflict of interest statement None}

Open Access This article is distributed under the terms of the Creative Commons Attribution Noncommercial License which permits any noncommercial use, distribution, and reproduction in any medium, provided the original author(s) and source are credited.

\section{References}

1. Jemal A, Siegel R, Ward E, Hao Y, Xu J, Murray T, Thun MJ (2008) Cancer statistics, 2008. CA Cancer J Clin 58(2):71-96

2. Friedlander M, Grogan M (2002) U.S. Preventative Services Task Force. Guidelines for the treatment of recurrent and metastatic cervical cancer. Oncologist 7(4):342-347

3. Ferrandina G, Legge F, Fagotti A, Fanfani F, Distefano M, Morganti A, Cellini N, Scambia G, 1 (Suppl 1) (2007) Preoperative concomitant chemoradiotherapy in locally advanced cervical cancer: safety, outcome, and prognostic measures. Gynecol Oncol 107(1 Suppl 1):S127-S132

4. Fulcher AS, O’Sullivan SG, Segreti EM, Kavanagh B (1999) Recurrent cervical carcinoma: typical and atypical manifestations. Radiographics 19:S103-S116

5. Matsuyama T, Tsukamoto N, Imachi M, Nakano H (1989) Bone metastasis from cervix cancer. Gynecol Oncol 32(1):72-75

6. Pasricha R, Tiwari A, Aggarwal T, Lal P (2006) Carcinoma of uterine cervix with isolated metastasis to fibula and its unusual behavior: report of a case and review of literature. J Cancer Res Ther 2(2):79-81

7. Moore DH, Blessing JA, McQuellon RP, Thaler HT, Cella D, Benda J, Miller DS, Olt G, King S, Boggess JF, Rocereto TF (2004) Phase III study of cisplatin with or without paclitaxel in stage IV B, recurrent or persistent squamous cell carcinoma of the cervix: a gynecologic oncology group study. J Clin Oncol 22:3113-3119

8. National Comprehensive Cancer Network (NCCN) (2008) NCCN clinical practice guidelines in oncology. http://www.ncen.org

9. Grigsby PW (2009) Role of PET in gynecologic malignancy. Curr Opin Oncol 21(5):420-424

10. Kidd EA, Siegel BA, Dehdashti F, Rader JS, Mutic S, Mutch DG, Powell MA, Grigsby PW (2009) Clinical outcomes of definitive intensity-modulated radiation therapy with fluorodeoxyglucosepositron emission tomography simulation in patients with locally advanced cervical cancer. Int $\mathbf{J}$ Radiat Oncol Biol Phys [Epub ahead of print] 